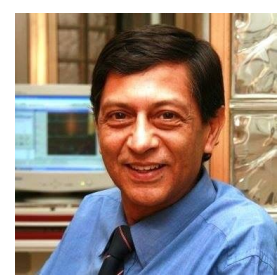

\title{
Peer Reviewing for Journals
}

Deepak Batura

The trouble with most of us is that we'd rather be ruined by praise than saved by criticism.

- Norman Vincent Peale

\section{Introduction}

The process of peer-review is not new. Perhaps, the earliest examples of professional peer review in medicine find mention in Arabic medical ethics texts of the 9th century, where physicians were encouraged to keep duplicate sets of notes. These notes could then be reviewed by a council of physicians to examine if treatment had been suitable and if there were lessons to be learned.

In the context of medical publishing, the process of having scholarly work scrutinised by other experts in the field before acceptance for publication began in the 17th century with Henry Oldenberg who founded the journal "Philosophical Transactions of the Royal Society". He solicited opinions of experts in the prepublication process. This process, known as scholarly peer review, has mostly remained unchanged to date.

\section{The aims of peer review}

Scholarly peer review seeks to subject an author's writings to a rigorous scientific inspection by other authorities in the field, to endorse the validity of the experimental work as seen from other experts' perspective and to preserve the robustness of the scientific process. The reviewer helps the chief editor in deciding the outcome of a scholarly submission and, as such, plays a large part in the journal's success and the paper's acceptance by the scientific community at large.

\section{An overview of the peer review process}

Once the journal office has received a paper, it is dealt with by an administrative editor who vets the article for its conformity to the journal's style and essential requirements such as conflicts of interests' disclaimers, disclosures of funding, ethical declarations and copyright forms. If these are in order, the manuscript makes its way to the desk of the chief editor or one of the section editors who examine the paper to see if it worthy of being reviewed. If suitable for review, it is sent out to appropriate reviewers. Once the reviews are back at the editor's desk, the editor studies the reviewers' reports and decides the outcome. If revisions are indicated, the process may need to be repeated once the author submits corrections. The steps in the publication process are at Fig 1.

\section{Types of peer-review}

Journals follow different models of peer review. The most common form of peer review is the single-blind peer review, where the reviewer knows the identity of the authors but not the other way around. However, being aware of the identity of the authors or their institution could lead to a bias on the reviewer's part.

DOI: $10.3126 / \mathrm{mjsbh} . v 18 \mathrm{i} 1.21868$

Submitted on: $2018-11-28$

Accepted on: 2018-12-26 


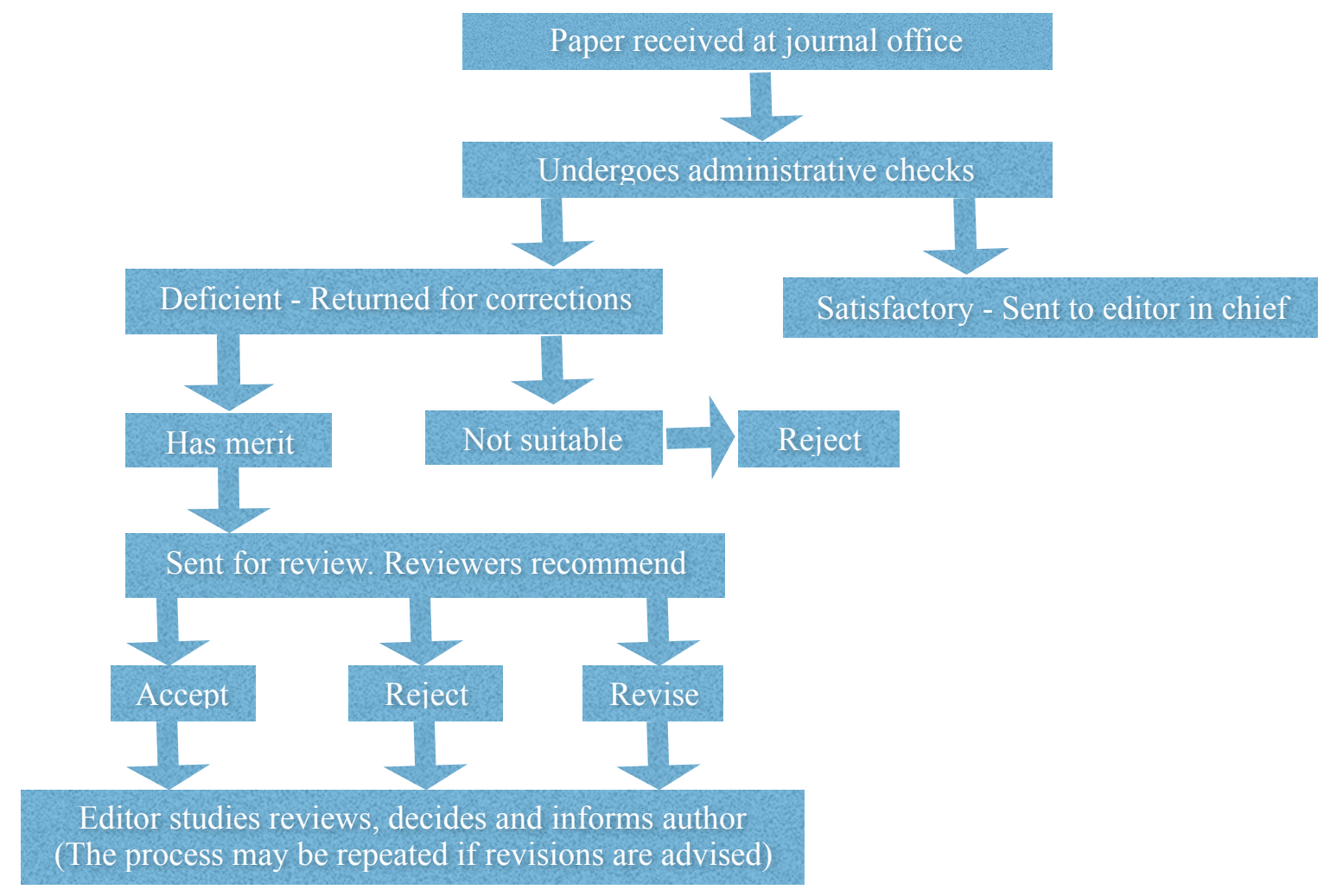

Fig 1. The peer review pathway

The weaknesses of a single-blind peer review are partially offset by the double-blind peer review in which neither the reviewer nor the authors are aware of the other's identity. While a double blind review potentially reduces bias, it could lead to irresponsible reviewing. Furthermore, in highly specialised fields, it may be possible to guess authorship. Nevertheless, many journals are now switching to this form of review because of a perceived higher objectivity. The Medical Journal of Shree Birendra Hospital follows this model of peer review.

Another type of review is the open type, where the identity of the authors and the reviewers are known to each other and the review is published alongside the paper, allowing readers to track the discourse and formulate their ideas about the scientific content and its criticism.

Finally, there is the relatively recent and yet mostly experimental post-publication review, where reviewers comments are published on the science in the paper after it has been published, leading to greater transparency, and hence greater accountability and ownership.

\section{What is expected of a reviewer?}

In the first place, a reviewer should not accept to review a manuscript if it is not within their sphere of competence or knowledge or if they do not have the time on the present occasion. Editors would appreciate an honest declaration to this effect as it allows them to find another reviewer whose field of interest might be more congruent with the content of the manuscript.

The reviewer should decide beforehand if there are any conflicts of interest in reviewing a particular paper. Examples of conflicts of interest include reviewing for authors whom they have worked with recently or having received sponsorship from a pharmaceutical company related to the products covered in the paper. Similarly, reviewers are expected to put aside personal equations and emotions before they accept an assignment. In the event they feel they might not be objective enough, it is honest and appropriate to declare an inability to review.

It is ethically incumbent on a reviewer to not share the contents of the paper with others or to plagiarise ideas from the articles they have been asked to review. Similarly, it is necessary to be dispassionate and polite 
while writing the review. Avoiding personal or offensively negative comments is imperative. A good rule of thumb is for the reviewer to put himself in the shoes of the author and reflect on how it would feel to receive a similarly worded comment on a paper they had authored. It is possible to be decisive without being impolite, and this is the hallmark of a good reviewer.

It is useful to remember that the chief role of the reviewer is to guide the editor in chief towards making a decision. Hence the review report must be clear, logical and unambiguous.

\section{How to carry out a useful peer-review}

The reviewer must carefully read the title, abstract and keywords to see if these accurately reflect what the study is attempting to demonstrate. Importantly, the abstract should not contain material that is not derived from the main text. As an abstract is often the only part of the paper that is read, it should include sufficient information to interest the reader, within the word limit specified by the journal.

The introduction should state the background leading to the research including salient references, the research hypothesis and the objectives of the study.

When reviewing the methods section, the reviewer must inspect if the section clarifies all the steps of the experiment, which should be replicable. For, example, specifications of the equipment and reagents used must be mentioned. Have controls been specified, are they appropriate and if the data collected is relevant to the objectives must be assessed. Finally, the statistical methods should apply to the data. If a reviewer is uncertain about the appropriateness of the statistical techniques, he should clarify this in his report so that the editor may seek an appropriate statistical review.

Analysing the results is of crucial importance as these form the basis of the arguments the authors are expected to advance in the discussion. Where the results are in a tabular form, the reviewer must scrutinise them as quite often errors may occur in tables. Similarly, graphs and diagrams should be an accurate reflection of the results.

In the Discussion and Conclusion sections, reviewers must see if the results have been suitably interpreted and appropriately weighed and referenced to previous work. The authors should have explained the limitations of their study and drawn conclusions for future applications or further research.

\section{Conclusion}

Peer reviewers should enjoy the task and appreciate the honour in having contributed to scientific increments in their field. It is good to bear in mind Cicero's comment:

I criticise by creation, not by finding fault.

Dr Deepak Batura

Department of Urology

London North West University Healthcare NHS Trust Watford Road, Harrow, London, UK HA1 3UJ

Email: deepakbatura@nhs.net 